\title{
Spectroscopy of Hadrons with $b$ Quarks from Lattice NRQCD
}

\author{
A. Ali Khan ${ }^{\mathrm{a} *}$ \\ aphysics Department, The Ohio State University, Columbus, OH 43210, USA
}

Preliminary results from an extensive lattice calculation of the $B, B_{c}$, and $\Upsilon$ spectrum at quenched $\beta=6.0$ are presented. The study includes radially and orbitally excited mesons, and baryons containing $b$ quarks. The $b$ quarks are formulated using NRQCD; for light and $c$ quarks, a tadpole-improved clover action is used.

\section{INTRODUCTION}

Experimental data on excited $B$ mesons and $b$ baryons have begun to emerge just recently. One hopes that in the next years, these states will be firmly established and accurate data on their masses become available. For the $B_{c}$, experimental results are so far restricted to preliminary values for the ground state mass.

It is therefore of great interest to obtain predictions for the $B$ and $B_{c}$ spectrum from lattice QCD. This talk is a status report on a calculation using NRQCD for the $b$ quarks, and a tree level tadpole-improved clover action for the light and charm quarks. The simulation was done quenched at $\beta=6.0$ on a lattice volume $16^{3} \times 48$. The NRQCD action and the simulation parameters for the heavy-light physics are described in Ref. 11. Our two $\kappa$ values around the charm are 0.119 and 0.126 . All the results presented in this article are preliminary.

\section{THE HEAVY-LIGHT SPECTRUM}

For the $B$ spectrum results presented here we fix the lattice spacing from $M_{\rho}$ and obtain $a^{-1}=$ 1.92(7) GeV. Our determination of the averaged $u$ and $d$, and strange, quark masses is described in Ref. [1]. For our heavy-light meson mass we use the definition $M^{\prime}$ of Ref. [1]. We fix the $b$ quark mass in a slightly different way from that in Ref. [1]. Instead of using the pseudoscalar meson

\footnotetext{
${ }^{*}$ In collaboration with T. Bhattacharya, S. Collins, C. T. H. Davies, R. Gupta, C. Morningstar, J. Shigemitsu and J. Sloan. Present address: Center for Computational Physics, University of Tsukuba, Tsukuba, Ibaraki 305, Japan.
}

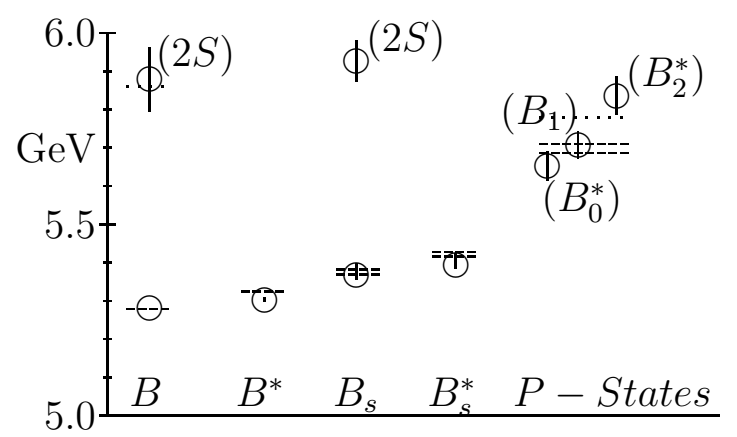

Figure 1. The $B$ meson spectrum. Dashed lines indicate experimental error bounds from the Particle Data Book, dotted lines, preliminary experimental results from DELPHI [2, 3].

mass, we set the spin-averaged meson mass to the physical value: $1 / 4\left(M_{B}+3 M_{B^{*}}\right)=5313 \mathrm{MeV}$.

\subsection{Mesons}

A summary of our results on the meson spectrum, compared with experimental results, is given in Fig. 1. The error bars include the statistical uncertainty, the error from interpolations and extrapolations to the physical quark masses, the statistical error in $a^{-1}$, and the uncertainty in fixing the strange quark mass. The figure shows that our calculation reproduces the presently known gross features of the $B$ meson spectrum.

Now we discuss the fine and hyperfine structure in more detail. For the $B^{*}-B$ splitting we obtain $24(5) \mathrm{MeV}$, which is significantly smaller 


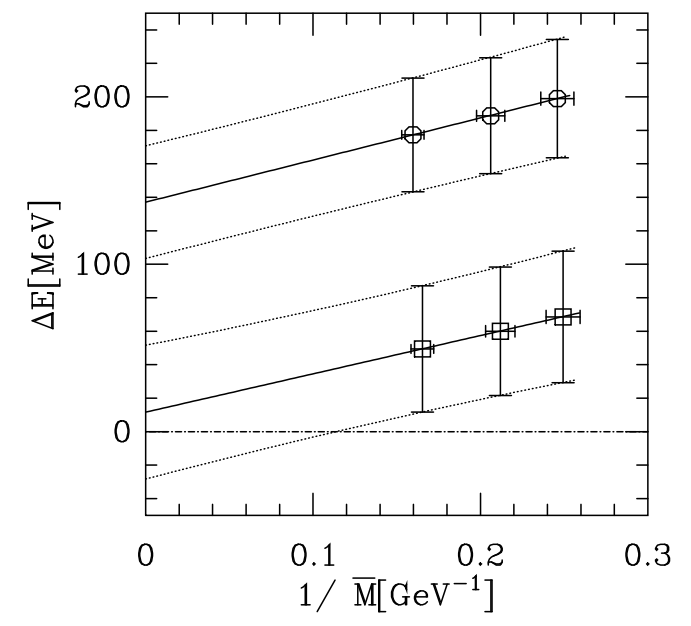

Figure 2. $\quad B_{2}^{*}-B_{0}^{*}$ (circles) and $B_{1}-B_{0}^{*}$ (squares) splittings as a function of the spinaveraged heavy-light meson mass $\bar{M}$. Solid lines denote a linear fit in $1 / \bar{M}$, dotted lines, the fit errors.

than the experimental value, 45.8(4) MeV. Possible sources of this discrepancy are quenching and discretization effects, corrections to the perturbative coefficient of the $\vec{\sigma} \cdot \vec{B}$ term in the action, and higher order relativistic corrections. For the $P$ state fine structure, the lattice provides predictions as there are no experimental numbers available. In heavy-light systems one differentiates $P$ states by the light quark angular momentum which can take the values $j_{l}=1 / 2$ and $j_{l}=3 / 2$. According to the coupling of the heavy quark spin, each of these levels splits up further into a hyperfine doublet; for $j_{l}=1 / 2$, this consists of $B_{0}^{*}$ and $B_{1}$, and for $j_{l}=3 / 2, B_{1}^{\prime}$ and $B_{2}^{*}$. The $B_{1}$ and the $B_{1}^{\prime}$ both have the quantum numbers $J^{P}=1^{+}$and can mix. Our two lattice operators for $J^{P}=1^{+}$give two slightly different masses. We assume that the lighter of our lattice results corresponds to the lighter physical state, which we expect to be the $B_{1}$. We were however not able to separate the contributions of the physical $1^{+}$states to the heavier lattice state.

In the following we consider the $B_{2}^{*}-B_{0}^{*}$ and

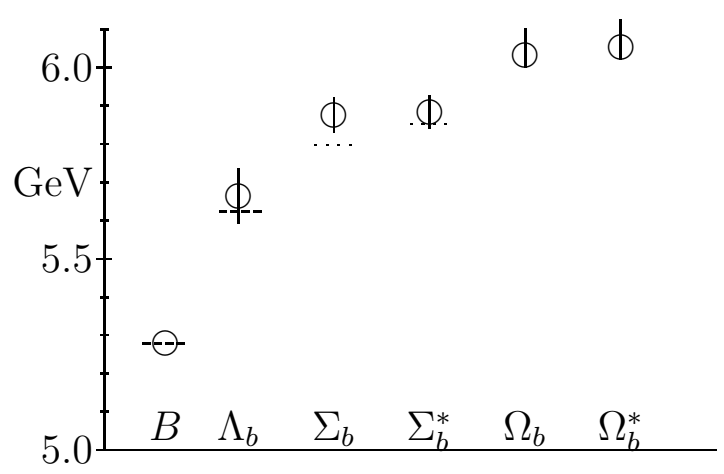

Figure 3. Baryons with one $b$ and two light quarks. Dashed lines indicate experimental error bounds from the Particle Data Book, dotted lines, preliminary experimental results from DELPHI [2].

the $B_{1}-B_{0}^{*}$ splitting. Both quantities, extrapolated to $\kappa_{l}$, are shown as a function of the inverse heavy mass in Fig. 2. According to our expectation, the $B_{2}^{*}-B_{0}^{*}$ is larger $(183(34) \mathrm{MeV})$ and has a finite static limit. The $B_{1}-B_{0}^{*}$ splitting is 54(38) $\mathrm{MeV}$, comparable to the $B^{*}-B$ splitting, and its static limit is compatible with zero.

\subsection{Baryons}

In heavy-light baryons with one $b$ quark, the two light quarks can couple to a sum spin $s_{l}=0$ or $s_{l}=1$. The former corresponds to the $\Lambda_{b}$ baryon, whereas the configuration with $s_{l}=1$ splits up into a hyperfine doublet consisting of the $\Sigma_{b}$ and the $\Sigma_{b}^{*}$. An overview of the results for baryons with one $b$ and two light quarks is shown in Fig. 3. The errors are calculated the in same way as for Fig. 1. The lattice results agree with experiment; the experimental values for the $\Sigma_{b}$ and the $\Sigma_{b}^{*}$ are however still preliminary.

The baryon hyperfine splitting $\Sigma_{b}^{*}-\Sigma_{b}$ as a function of the inverse heavy mass is presented in Fig. 1 . At the $b$ quark mass, we find 19(7) MeV. The extrapolation to infinite mass is compatible with zero. 


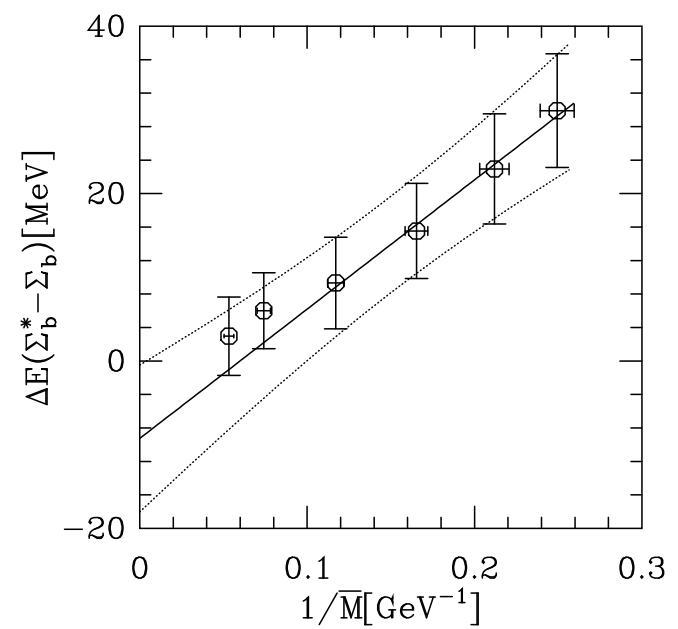

Figure 4 . The $\Sigma_{b}^{*}-\Sigma_{b}$ splitting, extrapolated to $\kappa_{l}$, as a function of the spin-averaged heavy-light meson mass $\bar{M}$. Solid lines denote a linear fit in $1 / \bar{M}$, dotted lines, the fit errors.

\section{THE $B_{c}$ SPECTRUM}

To minimize quenching errors, one ideally fixes the lattice spacing from a system that is sensitive to similar scales as the quantity that one is extracting. Here, we restrict ourselves to a presentation of the data quality and only a rough estimate of numbers, and do not perform a refined analysis. Since the $B_{c}$ is a heavy-heavy system, the charmonium $\bar{P}-\bar{S}$ splitting appears to be a reasonable choice. We use a preliminary number from Ref. [4] at $\beta=6.0, a^{-1} \sim 2.17 \mathrm{GeV}$. As $b$ and $c$ mass parameters for the $B_{c}$, we use $a M_{0}=2.0$ and $\kappa=0.126$. They correspond to physical $B$ and $\eta_{c}$ meson masses, respectively, at this lattice spacing.

A summary of the splittings between the excited states and the ground state is given in Fig. 5 .

The ground state mass is calculated in a similar way as for the $B$ :

$M\left(B_{c}\right)=E_{\text {sim }}\left(B_{c}\right)+\Delta_{\mathrm{NRQCD}}+\Delta_{\text {clover }}$.

The $\Delta$ 's are calculated from heavy-heavy NRQCD or clover systems respectively, at the

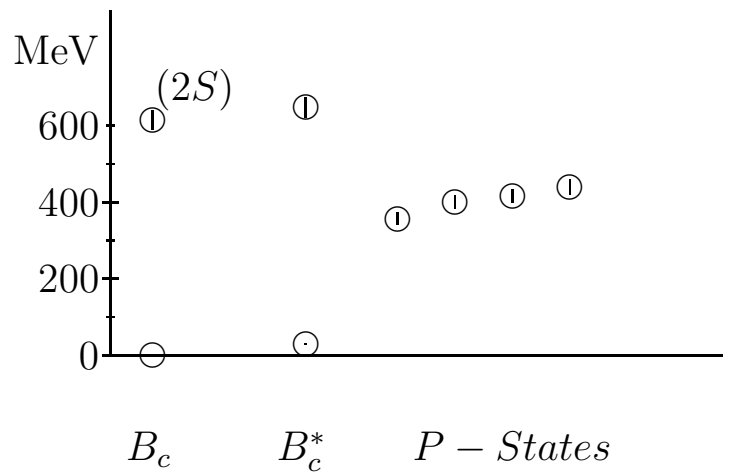

Figure $5 . B_{c}$ level splittings. The error bars are purely statistical.

same bare $b$ and $c$ quark masses as used for the $B_{c}$ :

$\Delta=1 / 2\left(M_{\text {kin }}(H H)-E_{\text {sim }}(H H)\right),$.

$E_{\text {sim }}$ is the falloff of the respective meson correlator, and for $M_{\text {kin }}$ we use the definition of Ref. [1]. We obtain $6.3(1) \mathrm{GeV}$, whereas $M_{\text {kin }}$ of the $B_{c}$ is $6.7(3) \mathrm{GeV}$. The difference is similar to the statistical error in $M_{\text {kin }}$, and is therefore not clearly significant. However it might be an indication of discretization effects expected in heavy-heavy mesons with clover quarks $[5]$. We are studying this further.

\section{Acknowledgements}

This work has been supported by the U.S. DOE. We thank the ACL at LANL and the NCSA at Urbana for computational support.

\section{REFERENCES}

1. A. Ali Khan et al., Phys. Lett. B 427 (1998) 132.

2. DELPHI Collaboration, DELPHI Note 95107, contribution to EPS '95.

3. DELPHI Collaboration, DELPHI 96-93 CONF 22, contribution to ICHEP ' 96.

4. P. Boyle, Ph.D. thesis, University of Edinburgh 1997.

5. A. Kronfeld, Nucl. Phys. B (Proc. Suppl.) 53 (1997) 401. 